\title{
Glycolytic pathway activity: effect on IVM and oxidative metabolism of bovine oocytes
}

\author{
Cynthia Gutnisky ${ }^{\mathrm{A}, \mathrm{C}}$, Sergio Morado ${ }^{\mathrm{A}}$, Gabriel C. Dalvit ${ }^{\mathrm{A}}$, \\ Jeremy G. Thompson ${ }^{\mathrm{B}}$ and Pablo D. Cetica ${ }^{\mathrm{A}}$ \\ A Institute of Research and Technology on Animal Reproduction, School of Veterinary Sciences, \\ University of Buenos Aires, Chorroarín 280, Buenos Aires C1427CWO, Argentina. \\ ${ }^{B}$ Research Centre for Reproductive Health, The Robinson Institute, School of Paediatrics \\ and Reproductive Health, The University of Adelaide, 2nd Floor, Medical School South, \\ Adelaide, SA 5005, Australia. \\ ${ }^{\mathrm{C}}$ Corresponding author. Email: cgutnisky@fvet.uba.ar
}

\begin{abstract}
The aim of the present study was to determine the effect of altering glycolytic pathway activity during bovine IVM on the meiotic maturation rate, oxidative activity, mitochondrial activity and the mitochondrial distribution within oocytes. Glycolytic activity was manipulated using two inhibitors (ATP, NaF) and a stimulator (AMP) of key enzymes of the pathway. Inhibition of glucose uptake, lactate production and meiotic maturation rates was observed when media were supplemented with ATP or NaF. The addition of AMP to the maturation medium had no effect on glucose uptake, lactate production or meiotic maturation. In the absence of gonadotrophin supplementation, AMP stimulated both glucose uptake and lactate production. However, AMP also decreased cytoplasmic maturation, as determined by early cleavage. During IVM, oocyte oxidative and mitochondrial activity was observed to increase at 15 and $22 \mathrm{~h}$ maturation. Inhibiting glycolysis with ATP or NaF led to a reduced oxidative and mitochondrial pattern compared with the respective control groups. Stimulation of the pathway with AMP increased oxidative and mitochondrial activity. A progressive mitochondrial migration to the central area was observed during maturation; oocytes treated with ATP, NaF or AMP showed limited migration. The present study reveals the effects of altering glycolytic pathway activity in cumulus-oocyte complexes, revealing the link between glycolysis of the cumulus-oocyte complex and the oxidative and mitochondrial activity of the oocyte.
\end{abstract}

Additional keywords: cumulus-oocyte complex, glycolysis, maturation, mitotracker green, redox sensor red.

Received 19 June 2012, accepted 20 September 2012, published online 26 October 2012

\section{Introduction}

Once the germinal vesicle $(\mathrm{GV})$ oocyte is released from the ovarian antral follicle, it will mature spontaneously in vitro under appropriate conditions. The maturation process that is manifested initially by GV breakdown (GVBD) also involves cumulus expansion and cytoplasmic maturation. In cattle oocytes, an adequate concentration of glucose in the maturation medium is necessary for this maturation process, as well as for subsequent embryo development (Rose-Hellekant et al. 1998; Lim et al. 1999; Khurana and Niemann 2000). Indeed, manipulation of glucose concentrations during maturation can affect the kinetics of bovine oocyte meiosis (Sutton-McDowall et al. 2005).

As in other cell types, cumulus-oocyte complexes (COCs) metabolise glucose via glycolysis, the pentose phosphate pathway and the hexosamine biosynthesis pathway (Downs and Utecht 1999; Sutton et al. 2003; Gutnisky et al. 2007), as well as the polyol pathway (for a review, see Sutton-McDowall et al.
2010). However, cumulus cells have a great capacity for glycolysis, and this represents the predominant pathway for glucose metabolism in COCs. It has been suggested that cumulus cell glycolytic activity is high in order to generate ATP and produce pyruvate, lactate, malate and/or oxalacetate, which are readily used as oxidative substrates by the oocyte (Brackett and Zuelke 1993; Cetica et al. 1999, 2002, 2003). In contrast, oocytes appear to lack the capacity to undertake significant glucose metabolism (Zuelke and Brackett 1992; Sutton et al. 2003; Dumollard et al. 2007). In agreement with these observations, cumulus cells express a high-affinity glucose transporter, namely Glut4 (Roberts et al. 2004), whereas the oocyte does not.

For mice, a high glucose concentration in the maturation medium inhibits maturation by increasing intracellular ATP levels (Downs and Mastropolo 1994). It has also been proposed that the metabolic activity of the oocyte increases in the latter half of the meiotic maturation process, coinciding with a rise in 
lactate production, which has been proposed as an indicator of oocyte quality (Preis et al. 2005).

In somatic cells, the major regulatory point of the glycolytic pathway is the enzyme phosphofructokinase 1 (EC 2.7.1.11), with AMP and ATP having important positive and negative allosteric regulating roles, respectively (Schirmer and Evans 1990; Clarenburg 1992). Sodium fluoride (NaF) is also a wellknown inhibitor of the pathway, inactivating the glycolytic enzyme enolase (EC 4.2.1.11; Mayes and Bender 2004). The intermediary metabolism of glucose also produces the reducing equivalent, NADH. This metabolite is mainly synthesised by cumulus cells in the glycolytic pathway by glyceraldehyde 3-phosphate dehydrogenase (GAPDH) and by the oocyte via the reaction catalysed by $\alpha$-ketoglutarate dehydrogenase and malate dehydrogenase. In addition to being used as a cofactor for anabolic pathways, NADH is a key redox molecule (Dumollard et al. 2007), important in both cytosolic and mitochondrial redox regulation. The redox state describes the sum of interactions between oxidised and reduced forms of a variety of molecules, including $\mathrm{NAD}(\mathrm{P})$ : $\mathrm{NAD}(\mathrm{P}) \mathrm{H}, \mathrm{FAD}: \mathrm{FADH}$, reduced glutathione: oxidised glutathione (for reviews, see Harvey et al. 2002; Herrick et al. 2006).

Oocyte maturation also includes cytoplasmic changes, such as the synthesis and accumulation of mRNA, proteins, transcription factors and the redistribution of organelles, especially mitochondria. Mitochondrial activity is essential for oocyte competence, and the ATP content of oocytes generated from the reducing equivalents derived from carboxylic acid metabolism through the tricarboxylic acid (TCA) cycle is highly correlated with oocyte competence (Stojkovic et al. 2001; Dumollard et al. 2007).

Mitochondrial distribution differs within in vitro maturing oocytes from different species. In mouse oocytes, mitochondria aggregate around the nucleus from the GV stage through to GVBD. With the movement of the nucleus throughout maturation, mitochondria concentrate around the nucleus (Nishi et al. 2003). In pig oocytes, mitochondria accumulate in the perinuclear area during meiotic progression from GVBD to anaphase I. In matured oocytes, mitochondrial foci are formed and move to the inner cytoplasm (Sun et al. 2001). Conversely, in bovine oocytes after $12-18 \mathrm{~h}$ IVM, the mitochondria move from a cortical location to an evenly distributed pattern (Hyttel et al. 1986). Furthermore, there appears to be an association between the ATP content of the oocyte and redistribution of the mitochondria; specifically, for oocytes with low ATP content, mitochondrial migration does not occur (Stojkovic et al. 2001).

Although it was proposed that glycolytic end products produced by cumulus cells are metabolised by the bovine oocyte during IVM, there are no studies that have evaluated the effect of glycolytic activity in COCs on oocyte oxidative metabolism and maturational capacity. Thus, the main aim of the present study was to investigate the glycolytic pathway activity during cattle oocyte IVM, analysing the effects of two inhibitors (ATP, NaF) and a stimulator (AMP) of the key enzymes of glycolysis on the maturation rate, oxidative and mitochondrial activity and mitochondrial distribution in oocytes.

\section{Materials and methods}

\section{Materials}

Unless specified otherwise, all chemicals and reagents were purchased from Sigma Chemical (St Louis, MO, USA).

\section{Recovery of COCs}

Bovine ovaries were collected at an abattoir within $30 \mathrm{~min}$ of death and kept warm $\left(30^{\circ} \mathrm{C}\right)$ during the 2-h journey to the laboratory. Ovaries were washed with physiological saline containing $100000 \mathrm{IU} \mathrm{L}^{-1}$ penicillin and $100 \mathrm{mg} \mathrm{L}^{-1}$ streptomycin. The COCs were recovered by aspiration of antral follicles $(2-5 \mathrm{~mm}$ in diameter) and only oocytes completely surrounded by a compact and multilayered cumulus oophorus were used.

\section{IVM of COCS}

The COCs were cultured in Medium 199 (Earle's salts, L-glutamine, $100 \mathrm{mg} \mathrm{L}^{-1}$; sodium bicarbonate, $2.2 \mathrm{mg} \mathrm{L}^{-1}$; GIBCO, Grand Island, NY, USA) supplemented with $5 \%(\mathrm{v} / \mathrm{v})$ fetal bovine serum (FBS; GIBCO), $0.2 \mathrm{mg} \mathrm{L}^{-1}$ porcine FSH (Folltropin-V; Bioniche, Belleville, Ontario, Canada), $2 \mathrm{mg} \mathrm{L}^{-1}$ porcine LH (Lutropin-V; Bioniche) and $50 \mathrm{mg} \mathrm{L}^{-1}$ gentamicin sulfate under mineral oil at $39^{\circ} \mathrm{C}$ for $22 \mathrm{~h}$ in an atmosphere of humidified $5 \% \mathrm{CO}_{2}$ in air.

To study the effects of different enzyme modulators of glycolysis on COC glycolytic pathway activity and oocyte meiotic maturation, COCs were cultured individually in $20-\mu \mathrm{L}$ drops of maturation medium supplemented with increasing concentrations of ATP $(1,10,20$ and $40 \mathrm{mM}), \mathrm{NaF}(2,3,4$ and $5 \mathrm{mM})$ or AMP $(1,10,20$ and $40 \mathrm{mM})$ under the conditions described above.

To investigate the effects of manipulating COC glycolytic pathway activity on subsequent oxidative activity, mitochondrial activity, mitochondrial distribution and oocyte nuclear morphology in oocytes, COCs were cultured in groups of 50 in $500-\mu \mathrm{L}$ drops of maturation medium supplemented with $10 \mathrm{mM}$ ATP or $3 \mathrm{mM} \mathrm{NaF}$ (determined as inhibitory concentrations in the previous experiments) for 9,15 and $22 \mathrm{~h}$, and $10 \mathrm{mM}$ AMP (determined as a stimulatory concentration in the previous experiment) without gonadotrophin supplementation. These maturations time-points were chosen for being consecutives to key events of the maturation process, namely GVBD, MI and extrusion of the first polar body, respectively (Fleming and Saacke 1972; Kruip et al. 1983; Gordon 1994).

\section{Determination of the glycolytic activity of COCs}

The COCs were removed from each $20-\mu \mathrm{L}$ drop and the glucose content was determined from the spent maturation medium. Glucose concentrations were determined using a spectrophotometric assay based on the oxidation of glucose by glucose oxidase and subsequent production of hydrogen peroxide (Trinder 1969). Positive controls comprising 20- $\mu \mathrm{L}$ drops of maturation medium were included in each experiment.

Lactate production in the culture medium was determined in the same droplets used to determine glucose uptake. Lactate production was measured using a spectrophotometric assay 
based on the oxidation of lactate and the subsequent production of hydrogen peroxide (Trinder 1969; Barham and Trinder 1972).

\section{Evaluation of oocyte meiotic maturation}

The COCs removed from each $20-\mu \mathrm{L}$ drop were used to evaluate meiotic maturation rates. Oocytes were denuded mechanically by repeated pipetting in phosphate-buffered saline (PBS) with $1 \mathrm{~g} \mathrm{~L}^{-1}$ hyaluronidase. Denuded oocytes were placed in a hypotonic medium of $2.9 \mathrm{mmol} \mathrm{L}^{-1}$ sodium citrate at $37^{\circ} \mathrm{C}$ for $15 \mathrm{~min}$, fixed on a slide with $3: 1$ ethanol:acetic acid (Tarkowski 1966), stained with 5\% (v/v) Giemsa (Merck, Darmstadt, Germany) for $15 \mathrm{~min}$ and observed under a light microscope at $\times 100$ and $\times 400$ magnification. Oocytes were considered mature when an MII chromosome configuration was evident.

\section{Evaluation of oocyte cytoplasmic maturation}

Immature COCs were divided into three groups for IVM in the medium described above (positive control), without supplementation of gonadotrophins (negative control) and only supplemented with $10 \mathrm{mM}$ AMP. After $21 \mathrm{~h}$ maturation, IVF was performed using frozen-thawed semen from a Holstein bull of proven fertility. The semen was thawed at $37^{\circ} \mathrm{C}$ in modified synthetic oviducal fluid (mSOF; Takahashi and First 1992), centrifuged twice at $500 \mathrm{~g}$ for $5 \mathrm{~min}$ and then resuspended in fertilisation medium to a final concentration of $2 \times 10^{9}$ motile spermatozoa $\mathrm{L}^{-1}$ after sperm selection by glass wool (Arzondo et al. 2012). Fertilisation was performed in IVF-mSOF, consisting of mSOF supplemented with $5 \mathrm{~g}$ bovine serum albumin (BSA) $\mathrm{L}^{-1}$ and $10000 \mathrm{U}_{\text {heparin }} \mathrm{L}^{-1}$, under mineral oil at $39^{\circ} \mathrm{C}$ in $5 \% \mathrm{CO}_{2}$ in air and $100 \%$ humidity for $20 \mathrm{~h}$. Zygotes were denuded by repeated pipetting and placed in $500 \mu \mathrm{L}$ in vitro culture (IVC)-mSOF, consisting of mSOF supplemented with $30 \mathrm{~mL}$ amino acid MEM L ${ }^{-1}$ (GIBCO), $10 \mathrm{~mL}$ non-essential amino acid MEM L ${ }^{-1}$ (GIBCO), 2 mmol L-glutamine $\mathrm{L}^{-1}$, $6 \mathrm{~g} \mathrm{~L}^{-1} \mathrm{BSA}$ and $5 \%(\mathrm{v} / \mathrm{v}) \mathrm{FBS}$ (GIBCO), under mineral oil at $39^{\circ} \mathrm{C}$ in $90 \% \mathrm{~N}_{2}: 5 \% \mathrm{CO}_{2}: 5 \% \mathrm{O}_{2}$ and $100 \%$ humidity for $24 \mathrm{~h}$. Cytoplasmic maturation was evaluated by the ratio of cleaved embryos. An additional cohort of 10 oocytes from each replicate was maintained through the fertilisation procedure without exposure to spermatozoa to test for parthenogenesis.

\section{Evaluation of oxidative and mitochondrial activity, as well as mitochondrial distribution}

From the total number of oocytes recovered, two-thirds were used to determine oxidative activity, mitochondrial activity and mitochondrial distribution after $0,9,15$ and $22 \mathrm{~h}$ maturation. These time-points were chosen because they correspond to key events of the maturation process, namely GVBD (6-8h), MI $(12-14 \mathrm{~h})$ and extrusion of the first polar body $(18-21 \mathrm{~h})$.

Cumulus cells were removed mechanically by repeated pipetting in PBS with $1 \mathrm{~g} \mathrm{~L}^{-1}$ hyaluronidase before the zona pellucida was dissolved with $5 \mathrm{~g} \mathrm{~L}^{-1}$ pronase for $1 \mathrm{~min}$.

Fluorescent probes and confocal microscopy were used to analyse the parameters mentioned above. Dual staining with RedoxSensor red CC-1 (Molecular Probes, Eugene, OR, USA) and MitoTracker green FM (Molecular Probes) were used in this experiment. Oocytes were coincubated with final concentrations of $1 \mathrm{nM}$ RedoxSensor red CC- 1 and $0.5 \mathrm{nM}$ MitoTracker green $\mathrm{FM}$ for $30 \mathrm{~min}$ at $37^{\circ} \mathrm{C}$ in the dark and then washed twice with PBS. Stained oocytes were then placed between a slide and coverslip for observation under a laser confocal microscope (Nikon C1 confocal scanning head, Nikon TE2000E; Nikon, Kanagawa, Japan). One optical section was examined for each oocyte. The images obtained were saved and then analysed using Adobe Photoshop CS2 (version 9; Adobe Systems Inc.)

Both red and green fluorescence emission intensities were determined in four different regions (squares) within three areas (i.e. Areas 1-3) of the oocyte, as shown in Fig. $1 a$ (Wakefield et al. 2007).

Oxidative activity was calculated as the sum of the average red fluorescence intensity in the three areas of the same oocyte. Mitochondrial activity was calculated as the sum of the average green fluorescence intensity in the three areas of the same oocyte. Ratios of green fluorescence intensity between Areas 3 and 1 were then calculated to compare the distribution of active mitochondria.

\section{Evaluation of oocyte nuclear morphology}

The remaining one-third of oocytes were used to evaluate nuclear morphology after $0,9,15$ and $22 \mathrm{~h}$ maturation. Denuded oocytes were fixed in a $40 \mathrm{mg} \mathrm{L}^{-1}$ paraformaldehyde solution for $1 \mathrm{~h}$ and then incubated in a permeabilising solution for $1.5 \mathrm{~h}$. Finally, the fixed oocytes were stained with $10 \mathrm{mg} \mathrm{L}^{-1}$ Hoechst 33342 solution for $15 \mathrm{~min}$.

Oocyte nuclear status was observed at a magnification of $\times 400$ under a Jenamed II epifluorescence microscope (Carl Zeiss Jena, Buenos Aires, Argentina) with a $410-\mathrm{nm}$ filter at each time-point.

\section{Statistical analysis}

The results of glucose uptake, lactate production, oxidative activity, mitochondrial activity and the ratio of green fluorescence intensity between Areas 3 and 1 to evaluate mitochondrial distribution are expressed as the mean \pm s.e.m. In studies evaluating glucose uptake and lactate production, comparisons were made by ANOVA followed by Bonferroni's post test. Oxidative activity, mitochondrial activity and mitochondrial distribution were compared using a $2 \times 4$ or $3 \times 4$ factorial design. Meiotic and cytoplasmic maturation rates between treatments were compared using Chi-squared analysis for nonparametric data. In all tests, significance was set at $P<0.05$.

\section{Results}

\section{Glycolytic activity of COCs and oocyte maturation}

To investigate the glycolytic pathway, COCs were incubated during maturation with increasing concentrations of pathway modulators. COCs matured in the presence of increasing concentrations of ATP exhibited dose-dependent inhibition of glucose uptake and lactate production $(P<0.05)$. A codependent inhibition on their progression to MII was also observed $(P<0.05$; Fig. $2 a)$.

The addition of increasing concentrations of $\mathrm{NaF}$ to the maturation medium produced a dose-dependent inhibition of 
(a)

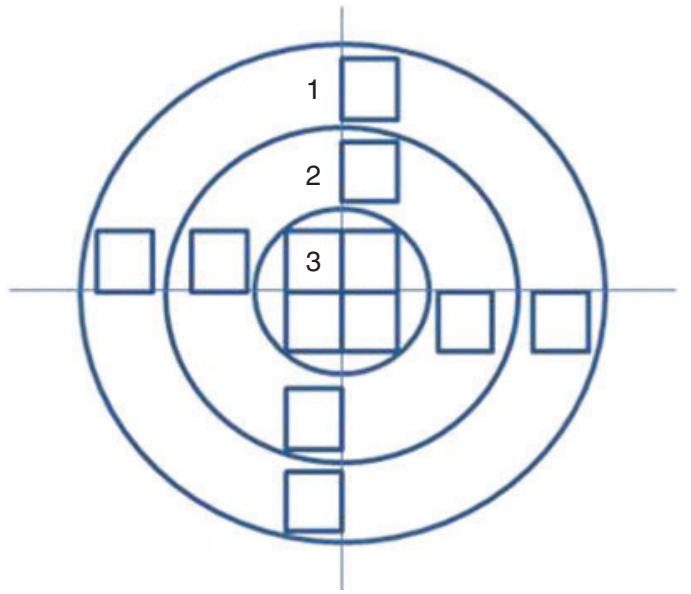

(b)
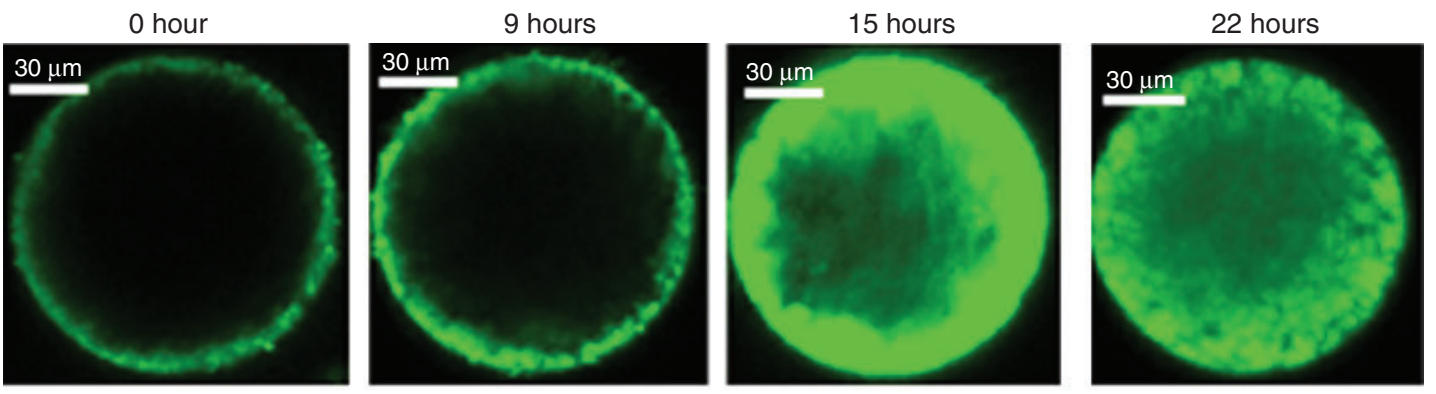

(c)
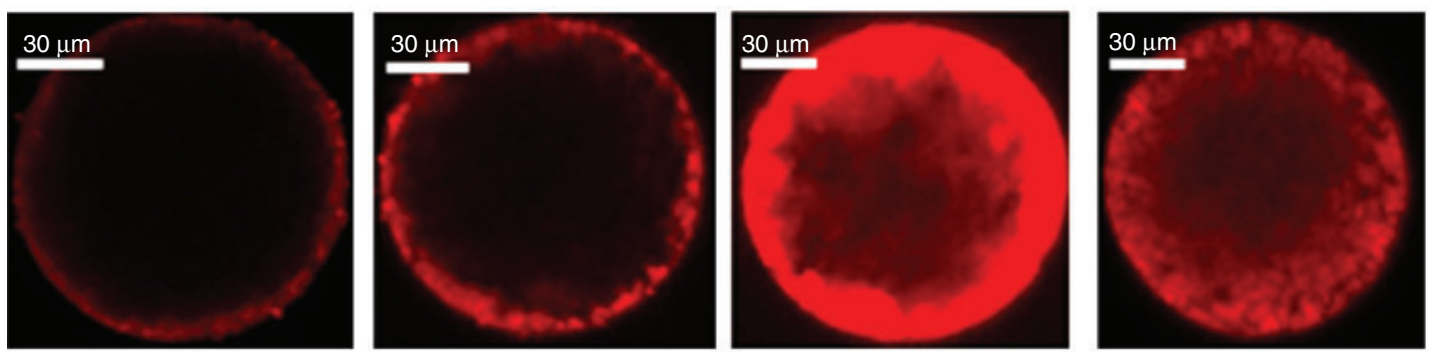

(d)
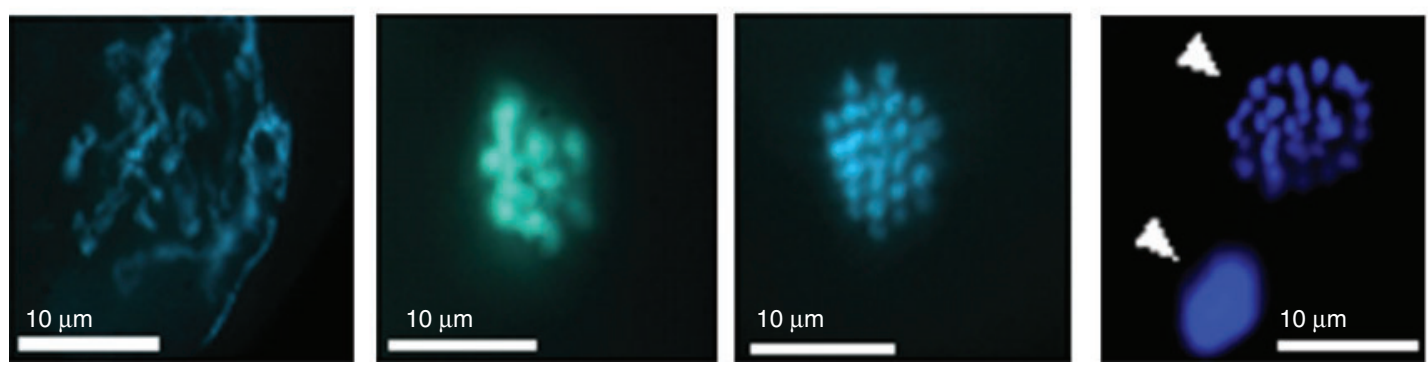

Fig. 1. (a) Schematic representation of the four different regions (squares) within three areas (Areas 1-3) of an oocyte used for determining mitochondrial fluorescence. $(b, c)$ Oocytes stained with two fluorescent probes, namely MitoTracker green FM (Molecular Probes, Eugene, OR, USA) (b) RedoxSensor red CC-1 (Molecular Probes) (c), after different times of maturation. ( $d$ ) Oocytes were stained with Hoechst DNA fluorochrome to determine nuclear maturation at different time points. Hour 0, germinal vesicle (GV) stage; Hour 9, GV breakdown; Hour 15, MI, Hour 22, extrusion of the first polar body. Arrows indicate the presence of an MII chromatin configuration and the first polar body.

both lactate production and nuclear maturation $(P<0.05)$. However, the inhibition of glucose uptake was not dose dependent $(P<0.05$; Fig. $2 b)$.

The addition of AMP to the maturation medium had no effect on glucose uptake and lactate production or on rates of nuclear maturation compared with the control group (Fig. 2c). To test whether the gonadotrophins (FSH, LH) were masking an effect of AMP, the experiment was repeated with maturation media devoid of gonadotrophins. In the absence of FSH and LH, AMP had stimulatory effects on both glucose uptake and lactate 

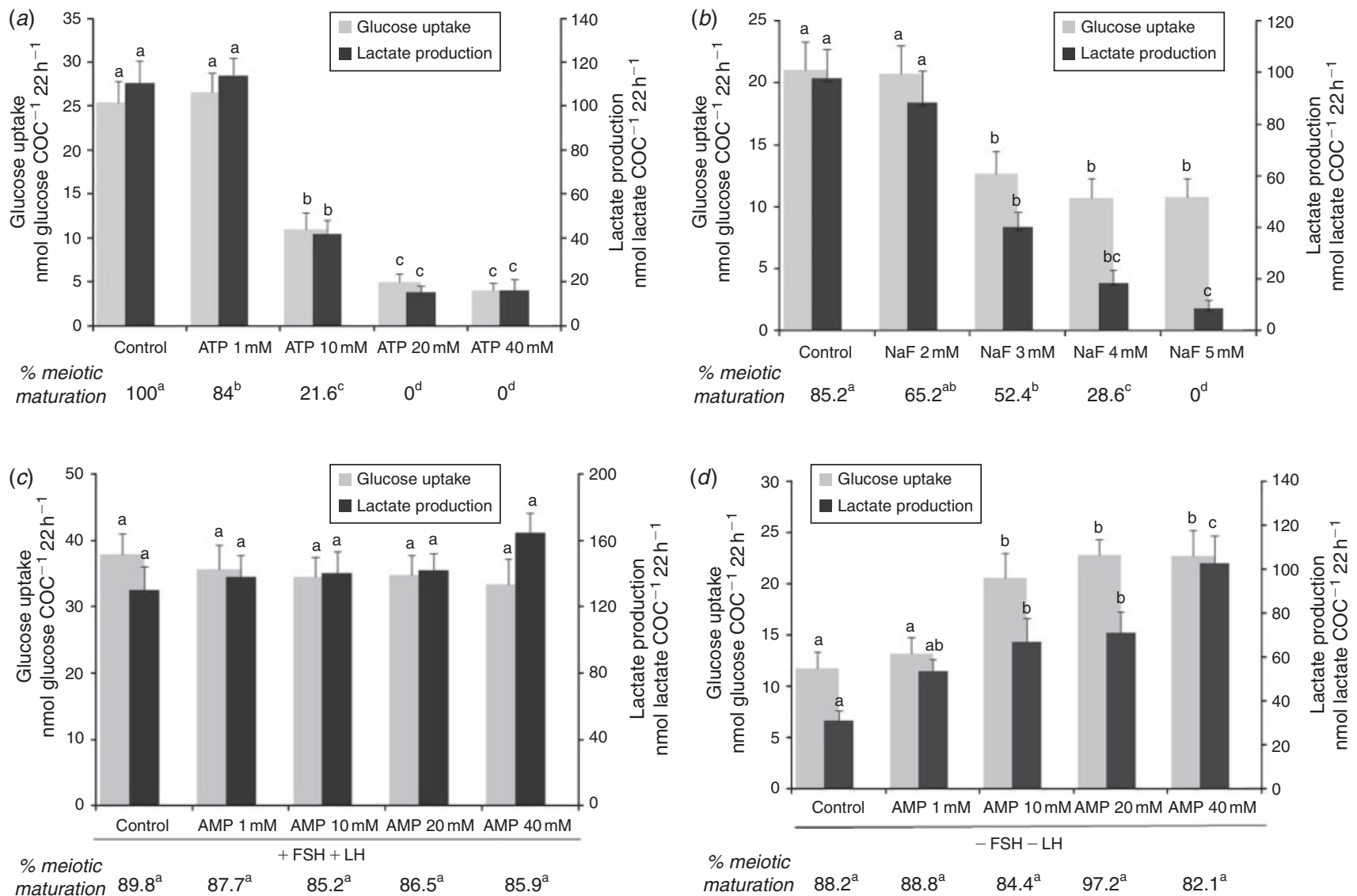

Fig. 2. Glucose uptake and lactate production in cumulus-oocyte complexes (COCs) matured for $22 \mathrm{~h}$ in the presence of different concentrations of ( $a$ ) ATP, $(b) \mathrm{NaF}$ and $(c, d)$ AMP with $(c)$ or without $(d)$ gonadotrophin supplementation. Data are the mean \pm s.e.m. $(n=30-40$ COCs for each treatment). Values for glucose uptake or lactate production with different superscript letters differ significantly $(P<0.05)$.

production $(P<0.05)$, with no effect on nuclear maturation (Fig. 2d).

Because the rate of nuclear maturation had not been altered with the addition of AMP, we evaluated the cytoplasmic maturation of oocytes cultured with $10 \mathrm{mM}$ AMP without gonadotrophin supplementation. We chose this concentration because it was the lowest that stimulated the glycolytic pathway in COCs. A significant decrease in cleavage rates compared with both control groups (matured with and without gonadotrophins) was observed in the group of COCs matured in the presence of AMP $(P<0.05$; Fig. 3$)$.

\section{Oxidative activity of the oocyte}

To confirm the participation of COC glycolysis in oxidative metabolism, oocytes were stained with RedoxSensor red CC-1 to quantify mitochondrial oxidative activity at different timepoints $(0,9,15$ and $22 \mathrm{~h})$. In these experiments, $10 \mathrm{mM} \mathrm{ATP}$ and $3 \mathrm{mM} \mathrm{NaF}$ were at the minimum inhibitory concentrations observed for glucose uptake and lactate production. For glycolytic pathway stimulation, we used $10 \mathrm{mM}$ AMP to stimulate glucose uptake and lactate production.

Oocytes exhibited changes in their oxidative activity throughout maturation, with an increase in oxidative activity

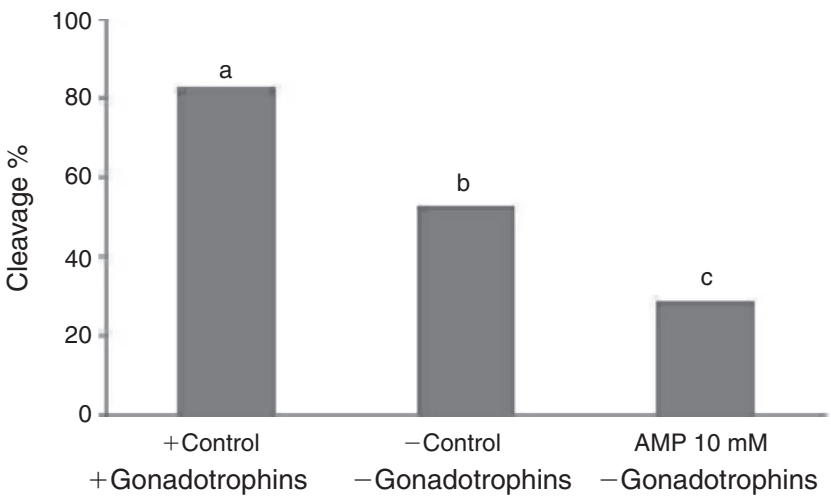

Fig. 3. Cleavage rate following IVF of oocytes matured for $22 \mathrm{~h}$ in the presence of $10 \mathrm{mM}$ AMP without gonadotrophins. +Control, positive control; - Control, negative control. Data are the mean \pm s.e.m. $(n=101-$ 116 COCs for each treatment). Values with different superscript letters differ significantly $(P<0.05)$.

observed after 15 and $22 \mathrm{~h}$ maturation compared with Time 0 $(P<0.05)$. Although similar changes in oxidative activity were observed in oocytes matured in the presence of ATP or NaF, overall activity was significantly lower than the values obtained 


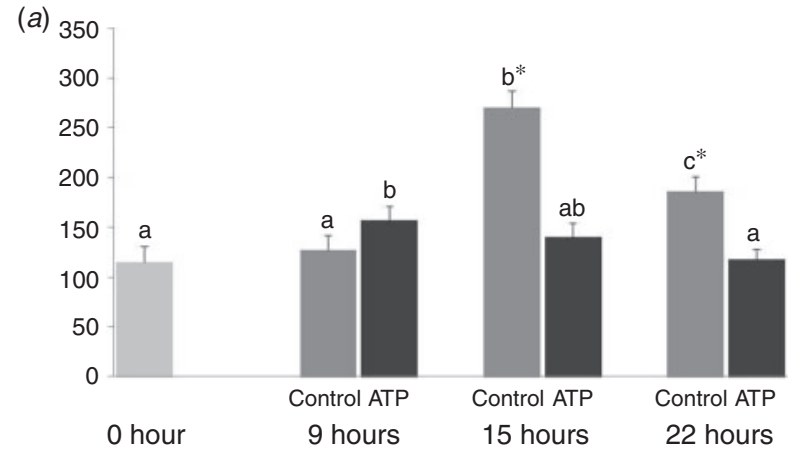

(b)

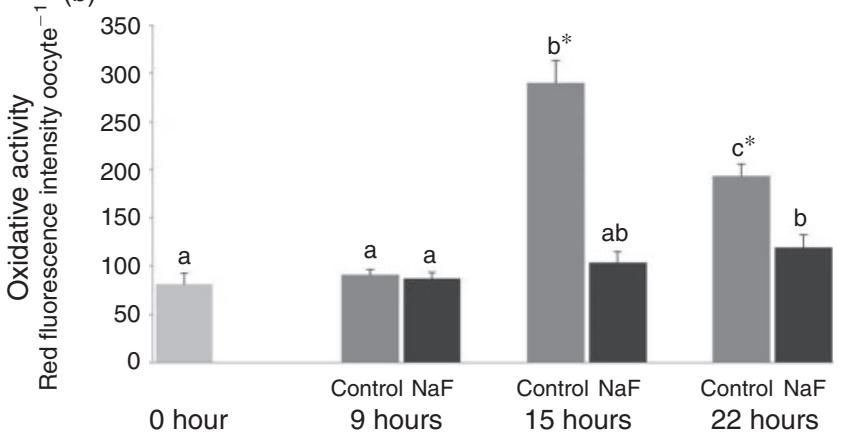

$(c) 350$

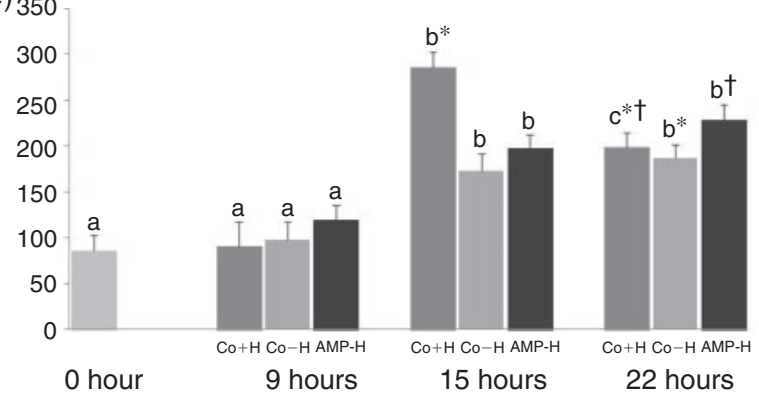

Fig. 4. Oxidative activity within oocytes matured for $0-22 \mathrm{~h}$ in the presence of (a) $10 \mathrm{mM}$ ATP, (b) $3 \mathrm{mM} \mathrm{NaF}$ or $(c) 10 \mathrm{mM}$ AMP. Data are the mean \pm s.e.m. ( $n=30-40$ COCs for each treatment). Within a graph and within each group (control or treated), values with different superscript letters differ significantly $(P<0.05) . * \dagger$ Columns with different symbols at the same time point differ significantly $(P<0.05)$.

for the control group $(P<0.05 ;$ Fig. $4 a, b)$. In contrast, AMP (in the absence of gonadotrophins) stimulated oxidative activity after 15 and $22 \mathrm{~h}$ maturation $(P<0.05)$ compared with Time 0. Nevertheless, after $15 \mathrm{~h}$ maturation, the increase was significantly lower for oocytes matured with either AMP or the control without gonadotrophins compared with the FSH + LH control $(P<0.05)$. In addition, oocytes matured with AMP showed the highest oxidative activity after $22 \mathrm{~h}$ maturation $(P<0.05$; Fig. 4c).

\section{Mitochondrial activity of oocytes}

To study the effects of manipulating glycolytic activity on mitochondrial activity within oocytes, the fluorescence intensity of MitoTracker green FM was analysed at the same time-points
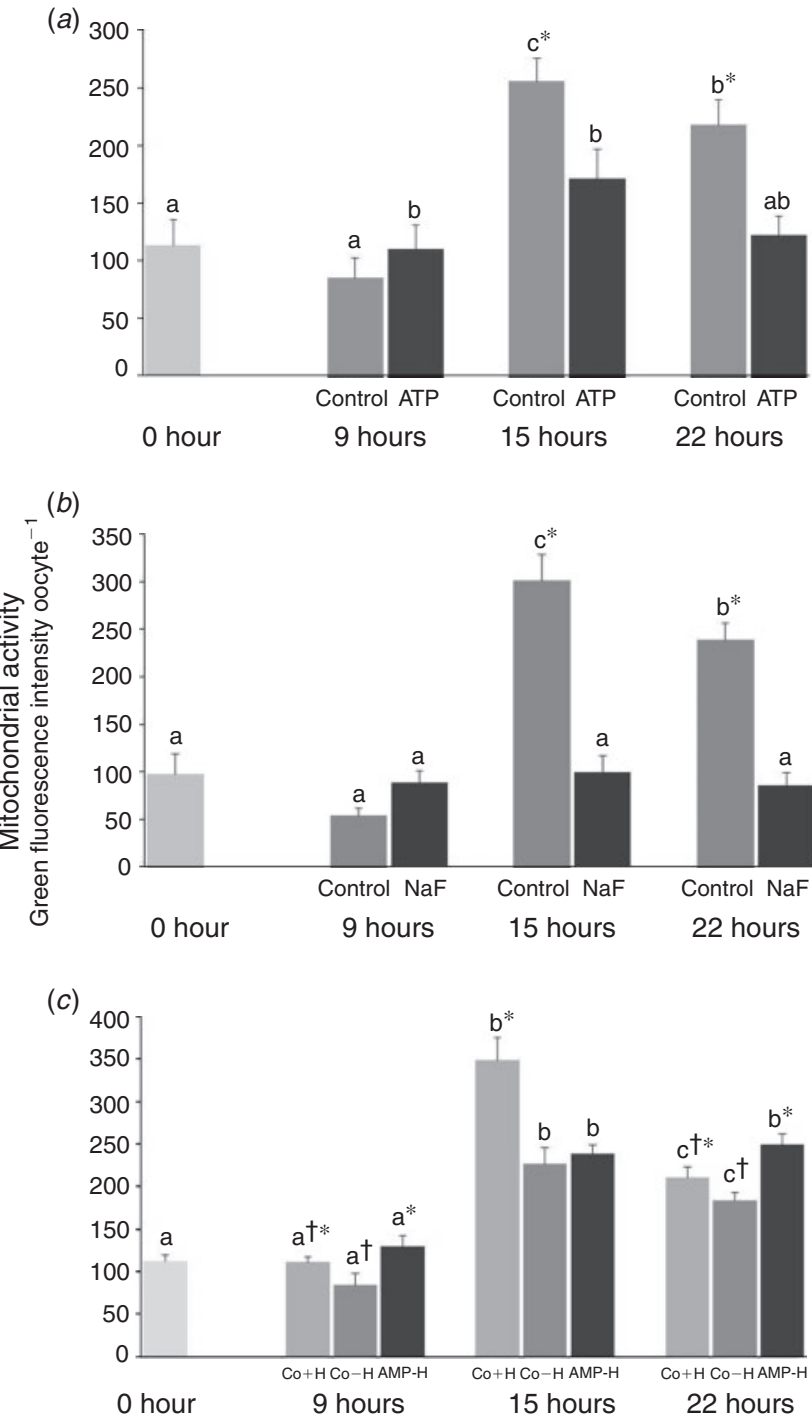

Fig. 5. Mitochondrial activity within oocytes matured for $0-22 \mathrm{~h}$ in the presence of (a) $10 \mathrm{mM}$ ATP, (b) $3 \mathrm{mM} \mathrm{NaF}$ or (c) $10 \mathrm{mM}$ AMP. Data are the mean \pm s.e.m. $(n=30-40$ COCs for each treatment). Within a graph and within each group (control or treated), values with different superscript letters differ significantly $(P<0.05)$. ${ }^{*}$ Columns with different symbols at the same time point differ significantly $(P<0.05)$.

and using the same ATP, NaF and AMP concentrations described for the experiment above.

The intensity of green fluorescence varied throughout maturation. A significant increase in intensity was observed after 15 and $22 \mathrm{~h}$ maturation compared with Time $0(P<0.05)$. An increase was observed in the ATP group after $15 \mathrm{~h}$ maturation compared with Time $0(P<0.05)$ (Fig. 5a, $b)$. Stimulation of glycolysis with AMP revealed an increase in mitochondrial activity after 15 and $22 \mathrm{~h}$ maturation compared with Time 0 $(P<0.05)$, as well as an increase in the same parameter compared with the respective controls after 9 and $22 \mathrm{~h}$ maturation $(P<0.05$; Fig. $5 c)$. 
A significant positive correlation was observed between the oxidative activity and mitochondrial activity of oocytes for each treatment $(r>0.82, P<0.05 ; n=199-211)$.

\section{Mitochondrial distribution in the oocyte}

Changes in mitochondrial distribution were observed during maturation. The distribution in immature oocytes was cortical (Fig. 1); however, during maturation, progressive mitochondrial migration to the central area was observed (Fig. 1). This observation was confirmed analysing the ratio of the intensity of green fluorescence between Area 3 and Area 1 of the oocyte $(P<0.05)$. In contrast, oocytes treated with ATP, NaF or AMP showed partial migration compared with their respective controls $(P<0.05$; Fig. $6 a-c)$.

\section{Oocyte nuclear morphology}

To determine which stage of nuclear maturation is affected by inhibition of glycolysis, COCs were also analysed using the fluorochrome Hoechst 33342 at the time-points specified. In the control group, $70.8 \%$ of oocytes had passed GVBD after $9 \mathrm{~h}$ maturation, whereas the rates were significantly lower for oocytes matured with ATP or NaF $(P<0.05)$. The rates of oocytes in MI after $15 \mathrm{~h}$ maturation were also significantly lower for groups treated with ATP and $\mathrm{NaF}(P<0.05)$. Extrusion of the first polar body was observed in $81.5 \%$ of control oocytes after $22 \mathrm{~h}$ maturation, but this rate decreased significantly $(P<0.05)$ in the treated groups, with most oocytes arrested at the GV stage (Table 1). Oocytes treated with AMP did not exhibit any differences in MII rates after $22 \mathrm{~h}$ maturation compared with controls, so we did not include the analysis of oocyte nuclear morphology in this experiment.

\section{Discussion}

The present study describes the effects of the addition of glycolytic modulators during bovine oocyte IVM on glucose uptake and lactate production of COCs, as well as on the maturation rate, oxidative activity, mitochondrial activity and mitochondrial distribution of oocytes.

When ATP or NaF were added to the maturation medium, glucose uptake and lactate production were reduced, as was the meiotic maturation rate, suggesting a relationship between COC glycolytic activity and the nuclear maturation capacity of the oocyte. Although dose-dependent inhibition of lactate production was seen with both modulators, only COCs matured with ATP exhibited dose-dependent inhibition of glucose uptake; this difference may be related to differences in the intracellular mechanisms of action of the two compounds. $\mathrm{NaF}$ is a specific pharmacological inhibitor of the glycolytic pathway that acts by inhibiting the enzyme enolase, whereas ATP is a physiological modulator of phosphofructokinase 1 and other glucosedependent metabolic pathways. NaF and ATP exert their effects at different levels within the glycolytic pathway: ATP inhibits the pathway at the preparatory phase, whereas $\mathrm{NaF}$ acts at the pay-off phase (Nelson and Cox 2005). The later inhibition of glycolysis by $\mathrm{NaF}$ may allow a flux of intermediary metabolites to enter other pathways.
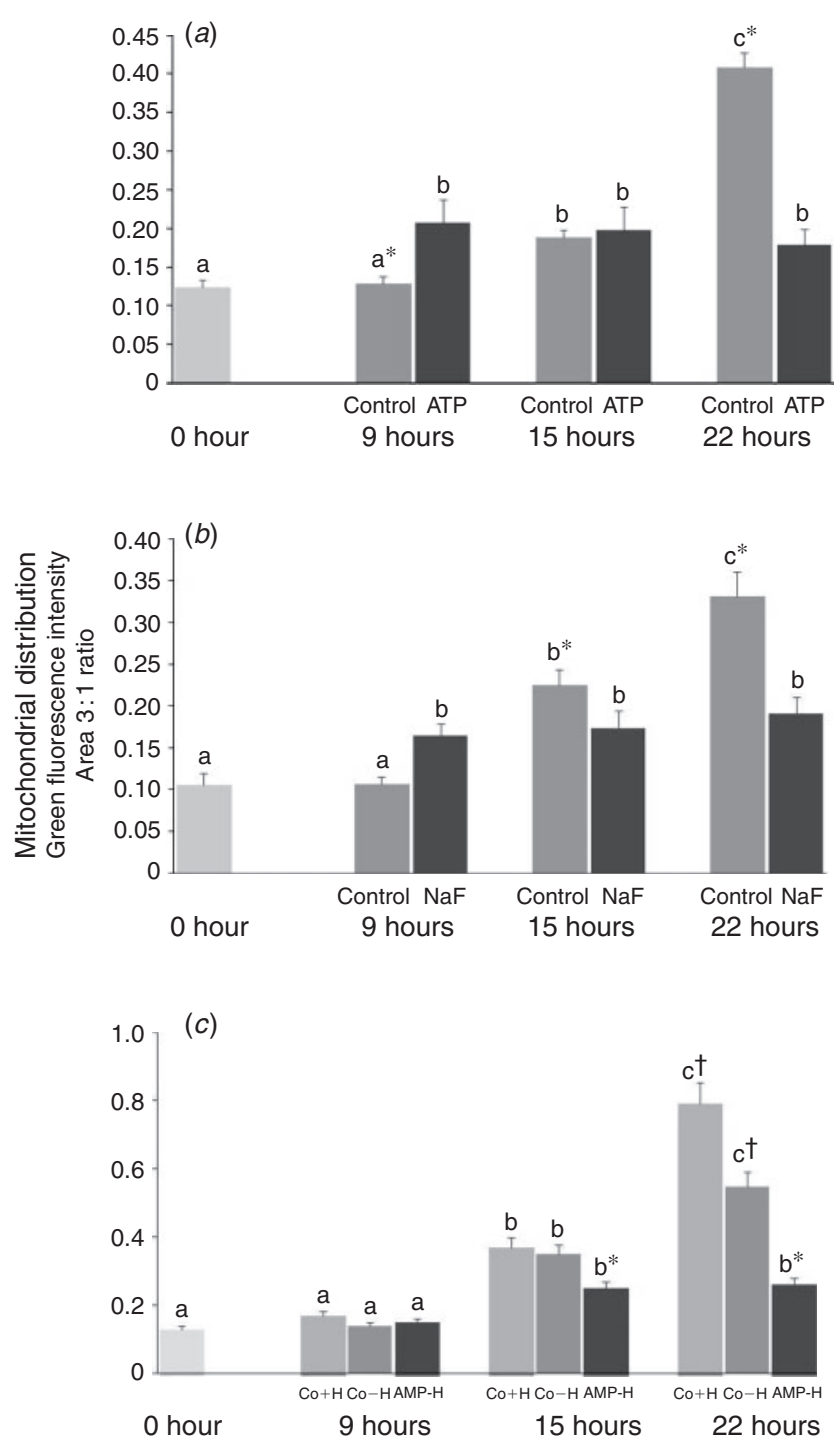

Fig. 6. Distribution of active mitochondria within oocytes matured for $0-22 \mathrm{~h}$ in the presence of (a) $10 \mathrm{mM} \mathrm{ATP},(b) 3 \mathrm{mM} \mathrm{NaF}$ or $(c) 10 \mathrm{mM}$ AMP. Data are the mean \pm s.e.m. $(n=30-40$ COCs for each treatment). Within a graph and within each group (control or treated), values with different superscript letters differ significantly $(P<0.05)$. ${ }^{*}$ Columns with different symbols at the same time point differ significantly $(P<0.05)$.

The dose-dependent inhibition of oocyte nuclear maturation in the presence of either ATP or NaF may be attributed a reduced contribution of oxidative substrates, such as pyruvate and lactate, by the cumulus cells. These substrates are essential for the bovine oocyte and the findings reflect the very low capacity for glucose metabolism by the oocyte (Cetica et al. 1999; Zuelke and Brackett 1992), as demonstrated by the considerably lower activity of phosphofructokinase 1 in denuded oocytes than in cumulus cells (Cetica et al. 2002).

In a previous study, we demonstrated that FSH + LH caused a significant $(30.5 \%)$ increase in glucose uptake by COCs (Gutnisky et al. 2007). In the present study, we found that the 
Table 1. Effects of $10 \mathrm{mM}$ ATP and $3 \mathrm{mM} \mathrm{NaF}$ on nuclear morphology

Data show the percentage of oocytes at each stage of development ( $n=15-20$ oocytes for each treatment). Different superscript letters indicates significant differences in the percentage of oocytes at the same time point and same nuclear stage between treatments. GV, germinal vesicle; GVBD, GV breakdown; PBE, polar body extrusion

\begin{tabular}{|c|c|c|c|c|c|c|c|c|c|c|c|c|}
\hline \multirow[t]{2}{*}{ Duration of maturation (h) } & \multicolumn{4}{|c|}{ Control } & \multicolumn{4}{|c|}{ ATP } & \multicolumn{4}{|c|}{$\mathrm{NaF}$} \\
\hline & GV & GVBD & MI & PBE & GV & GVBD & MI & PBE & GV & GVBD & MI & PBE \\
\hline 0 & $100^{\mathrm{a}}$ & & & & $100^{\mathrm{a}}$ & & & & $100^{\mathrm{a}}$ & & & \\
\hline 9 & $29.2^{\mathrm{a}}$ & $70.8^{\mathrm{a}}$ & & & $100^{\mathrm{b}}$ & $0^{\mathrm{b}}$ & & & $88.9^{\mathrm{b}}$ & $11.1^{\mathrm{b}}$ & & \\
\hline 15 & $9.5^{\mathrm{a}}$ & & $90.5^{\mathrm{a}}$ & & $87.5^{\mathrm{b}}$ & & $12.5^{\mathrm{b}}$ & & $84.2^{\mathrm{b}}$ & & $15.8^{\mathrm{b}}$ & \\
\hline 22 & $11.1^{\mathrm{a}}$ & & $7.4^{\mathrm{a}}$ & $81.5^{\mathrm{a}}$ & $100^{\mathrm{b}}$ & & $0^{\mathrm{a}}$ & $0^{\mathrm{b}}$ & $58.8^{\mathrm{c}}$ & & $17.7^{\mathrm{a}}$ & $23.5^{\mathrm{c}}$ \\
\hline
\end{tabular}

dose-dependent stimulation of glycolysis by AMP is not synergistic with FSH + LH stimulation. Furthermore, although the kinetics of meiotic maturation were not altered when AMP was added to the culture medium, the subsequent cleavage rates following IVF were significantly reduced, suggesting that stimulation of the glycolytic pathway by AMP may affect the cytoplasmic maturation of bovine oocytes. In line with these results, Preis et al. (2005) suggested that glucose uptake and lactate production by the COC are related to the capability of the oocyte to be fertilised. Moreover, AMP may be affecting the AMP : ATP ratio, resulting in the activation of AMP kinase. The activity of this enzyme is known to affect the nuclear maturation of bovine oocytes (Bilodeau-Goeseels et al. 2007).

In the present study, fluctuations in oxidative activity and mitochondrial activity of bovine oocytes were detected during IVM. In addition, both parameters were highly correlated and exhibited similar variations during maturation. Interestingly, the oxidative fluctuations observed here are not coincident with the changes in concentrations in reactive oxygen species detected during bovine oocyte maturation in vitro (Morado et al. 2009). Our results reveal that oxidative activity increases in bovine oocytes during maturation after 15 and $22 \mathrm{~h}$ culture. The increase in oxidative activity is coincident with key meiotic events of maturation, such as formation of the metaphase plate of the first meiotic division and extrusion of the first polar body (Gordon 1994), respectively. Protein synthesis increases threefold during MI compared with the GV stage (for a review, see Ferreira et al. 2009), suggesting that the increase in oxidative activity after $15 \mathrm{~h}$ maturation is related to new protein synthesis.

The reduced glycolytic activity induced by ATP and $\mathrm{NaF}$ is coincident with low oocyte oxidative activity after 15 and $22 \mathrm{~h}$ maturation, compared with untreated COCs. We also observed that inhibiting the glycolytic pathway arrested most of the oocytes at the GV stage. Although these results suggest that glycolytic activity of the COC may be decreased due to inhibition of GVBD and oocyte maturation, we have demonstrated previously that glycolytic activity in cumulus cells is not influenced by the presence of the oocyte (Sutton et al. 2003). Therefore, the pattern of oxidative activity would be dependent on changes in the glycolytic activity of the COCs and would therefore reflect ATP demand. Conversely, stimulation of the pathway by AMP affected the pattern of oocyte oxidative activity compared with the control group. The maintenance of a higher oxidative activity in the oocyte during IVM may, in some way, be affecting the cytoplasmic maturation of the female gamete.

Quantification of mitochondrial activity in untreated COCs increased significantly after 15 and $22 \mathrm{~h}$ maturation. Inhibition of COC glycolysis prevented this increase in mitochondrial activity, most likely as a consequence of the inhibition of glycolytic activity in COCs, resulting in a decreased supply of oxidative substrates to the oocyte. A similar observation was made for mitochondrial distribution: inhibition of glycolysis prevented mitochondrial migration during maturation. Stimulating the glycolytic pathway by AMP altered the pattern of mitochondrial activity compared with that in the control group. As discussed above with regard to oxidative activity, mitochondrial activity remained higher during IVM in the presence of AMP, suggesting that the increased glycolytic activity of cumulus cells may increase the contribution of reduced coenzymes and/or oxidative substrates to the oocyte, augmenting mitochondrial activity. Nevertheless, this does not appear to improve cytoplasmic maturation.

RedoxSensor red CC-1 (oxidative levels) and MitoTracker green FM (mitochondrial activity) were closely colocalised and within the cytosolic compartment of the oocyte, this being congruent with the characteristic pattern of metabolically active cells (Chen and Gee 2000). This relationship may be the result of an increase in cytosolic reductive agents, such as lactate and malate, within the oocyte. These metabolites can act as reductive compounds when they are substrates of lactate dehydrogenase and malate dehydrogenase, respectively (Cetica et al. 1999, 2003). It has been reported that mitochondrial reorganisation and a burst of ATP production during oocyte maturation are completely inhibited if cell cycle progression is inhibited ( $\mathrm{Yu}$ et al. 2010). In agreement with these findings, we determined that changes in oxidative and mitochondrial activity are related to modifications in normal mitochondrial migration, suggesting that the same is true in bovine oocytes.

In conclusion, we report herein that glycolytic pathway activity in COCs is necessary for successful IVM of the bovine oocyte. Modifications in the oxidative and mitochondrial activities in the oocyte are associated with increases at 15 and $22 \mathrm{~h}$ incubation during maturation. Inhibiting glycolysis reduced these parameters in the oocyte at these time-points, with an inhibition of mitochondrial migration in oocytes also detected. Stimulation of the glycolytic pathway by AMP in the absence of 
gonadotrophins also changed oxidative behaviour, which reduced oocyte cytoplasmic competence.

\section{Acknowledgements}

This work was supported by a grant from the University of Buenos Aires. The authors thank The Japanese International Cooperation Agency (JICA) for technology transfer and equipment, Deltacar Abattoir (Deltacar S.A. General Rodriguez, Bs. As., Argentina) for ovaries, Mr V. H. Chaves for recovering the ovaries and Astra Laboratories (Astra S.A., Haedo, Bs. As., Argentina) for ultrapure water.

\section{References}

Arzondo, M., Caballero, J., Marin Briggiler, C., Dalvit, G., Cetica, P., and Vazquez-Levin, M. (2012). Glass wool filtration of bull cryopreserved semen: a rapid, effective method to obtain a high percentage of functional spermatozoa. Theriogenology 78, 201-209. doi:10.1016/ J.THERIOGENOLOGY.2012.02.001

Barham, D., and Trinder, P. (1972). An improved colour reagent for the determination of glucose by the oxidase system. Analyst 97, 142-145. doi:10.1039/AN9729700142

Bilodeau-Goeseels, S., Sasseville, M., Guillemette, C., and Richard, F. (2007). Effects of adenosine monophosphate-activated kinase activators on bovine oocyte nuclear maturation in vitro. Mol. Reprod. Dev. 74, 1021-1034. doi:10.1002/MRD.20574

Brackett, B., and Zuelke, K. (1993). Analysis of factors involved in the in vitro production of bovine embryos. Theriogenology 39, 43-64. doi:10.1016/0093-691X(93)90023-X

Cetica, P., Pintos, L., Dalvit, G., and Beconi, M. (1999). Effect of lactate dehydrogenase activity and isoenzyme localization in bovine oocytes and utilization of oxidative substrates on in vitro maturation. Theriogenology 51, 541-550. doi:10.1016/S0093-691X(99)00008-4

Cetica, P., Pintos, L., Dalvit, G., and Beconi, M. (2002). Activity of key enzymes involved in glucose and triglyceride catabolism during bovine oocyte maturation in vitro. Reproduction 124, 675-681. doi:10.1530/ REP.0.1240675

Cetica, P., Pintos, L., Dalvit, G., and Beconi, M. (2003). Involvement of enzymes of amino acid metabolism and tricarboxylic acid cycle in bovine oocyte maturation in vitro. Reproduction 126, 753-763. doi:10.1530/REP.0.1260753

Chen, C., and Gee, K. (2000). Redox-dependent trafficking of 2,3,4,5,6- of pentafluorodihydrotetramethylrosamine, a novel fluorogenic indicator of cellular oxidative activity. Free Radic. Biol. Med. 28, 1266-1278. doi:10.1016/S0891-5849(00)00265-3

Clarenburg, R. (1992). Carbohydrate metabolism. In 'Physiological Chemistry of Domestic Animals'. (Ed. R. Reinhardt.) pp. 239-291. (Mosby Year Book: St Louis.)

Downs, S., and Mastropolo, A. (1994). The participation of energy substrates in the control of meiotic maturation in murine oocytes. Dev. Biol. 162 , 154-168. doi:10.1006/DBIO.1994.1075

Downs, S., and Utecht, A. (1999). Metabolism of radiolabeled glucose by mouse oocytes and oocyte-cumulus cell complexes. Biol. Reprod. 60 , 1446-1452. doi:10.1095/BIOLREPROD60.6.1446

Dumollard, R., Ward, Z., Carroll, J., and Duchen, M. (2007). Regulation of redox metabolism in the mouse oocyte and embryo. Development 134, 455-465. doi:10.1242/DEV.02744

Ferreira, E., Vireque, A., Adona, P., Meirelles, F., Ferriani, R., and Navarro, P. (2009). Cytoplasmic maturation of bovine oocytes: structural and biochemical modification and acquisition of developmental competence. Theriogenology 71, 836-848. doi:10.1016/J.THERIOGENOL OGY.2008.10.023

Fleming, W., and Saacke, R. (1972). Fine structure of the bovine oocytes and early embryos. J. Reprod. Fertil. 29, 203-213. doi:10.1530/JRF.0. 0290203
Gordon, I. (Ed.) (1994). Oocyte recovery and maturation. In 'Laboratory Production of Cattle Embryos'. pp. 31: 142. (Cab International: Wallingford.)

Gutnisky, C., Dalvit, G., Pintos, L., Thompson, J., Beconi, M., and Cetica, P. (2007). Influence of hyaluronic acid synthesis and cumulus mucification on bovine oocyte in vitro maturation, fertilisation and embryo development. Reprod. Fertil. Dev. 19, 488-497. doi:10.1071/RD06134

Harvey, A., Kind, K., and Thompson, J. (2002). Redox regulation of embryo development. Reproduction 123, 479-486. doi:10.1530/REP.0.1230479

Herrick, J., Brad, A., and Krisher, R. (2006). Chemical manipulation of glucose metabolism in porcine oocytes: effects on nuclear and cytoplasmatic maturation in vitro. Reproduction 131, 289-298. doi:10.1530/ REP.1.00835

Hyttel, P., Xu, K., Smith, S., and Greve, T. (1986). Ultrastructure of in vitro oocyte maturation in cattle. J. Reprod. Fertil. 78, 615-625. doi:10.1530 JRF.0.0780615

Khurana, N., and Niemann, H. (2000). Effect of oocyte quality, oxygen tension, embryo density, cumulus cells and energy substrates on cleavage and morula/blastocyst formation of bovine embryos. Theriogenology 54, 741-756. doi:10.1016/S0093-691X(00)00387-3

Kruip, T., Cran, D., Beneden, T., and Dieleman, S. (1983). Structural changes in bovine oocytes during final maturation in vivo. Gamete Res. 8, 29-47. doi:10.1002/MRD.1120080105

Lim, J., Lee, B., Lee, E., Chung, H., Ko, J., Park, S., Cha, K., and Hwang, W. (1999). In vitro maturation and in vitro fertilization of bovine oocytes cultured in a chemically defined, protein-free medium: effects of carbohydrates and amino acids. Reprod. Fertil. Dev. 11, 127-132. doi:10.1071/RD99001

Mayes, P., and Bender, D. (2004). Glucólisis y la oxidación del piruvato. In 'Harper. Bioquímica Ilustrada'. 16th edn. (Ed. R. K. Murray.) pp. 153-161. (Editorial El Manual Moderno: México DF.)

Morado, S., Cetica, P., Beconi, M., and Dalvit, G. (2009). Reactive oxygen species in bovine oocyte maturation in vitro. Reprod. Fertil. Dev. 21, 608-614. doi:10.1071/RD08198

Nelson, D., and Cox, M. (2005). Glycolysis, gluconeogenesis and the pentose phosphate pathway. In 'Lehninger. Principles of Biochemistry' 4th edn. (Eds K. Ahr and M. Ryan.) pp. 522-534. (W. H. Freeman and Co.: New York.)

Nishi, Y., Takeshita, T., Sato, K., and Araki, T. (2003). Change of the mithocondrial distribution in mouse ooplasm during in vitro maturation. J. Nippon Med. Sch. 70, 408-415. doi:10.1272/JNMS.70.408

Preis, K., Seidel, G., Jr, and Gardner, D. (2005). Metabolic markers of developmental competence for in vitro-matured oocytes. Reproduction 130, 475-483. doi:10.1530/REP.1.00831

Roberts, R., Stark, J., Iatropoulou, A., Becker, D., Franks, S., and Hardy, K. (2004). Energy substrate metabolism of mouse cumulus-oocyte complexes: response to follicle-stimulating hormone is mediated by the phosphatidylinositol 3-kinase pathway and is associated with oocyte maturation. Biol. Reprod. 71, 199-209. doi:10.1095/BIOLREPROD. 103.023549

Rose-Hellekant, T., Libersky-Williamson, E., and Bavister, B. (1998). Energy substrates and amino acids provided during in vitro maturation of bovine oocytes after acquisition of developmental competence. Zygote 6, 285-294. doi:10.1017/S0967199498000239

Schirmer, T., and Evans, P. (1990). Structural basis of the allosteric behaviour of phosphofructokinase. Nature 343, 140-145. doi:10.1038/ 343140A0

Stojkovic, M., Machado, S., Stojkovic, P., Zakhartchenko, V., Hutzler, P., Gonçalves, P., and Wolfi, E. (2001). Mitochondrial distribution and adenosine triphosphate content of bovine oocytes before and after in vitro maturation: correlation with morphological criteria and developmental capacity after in vitro fertilization and culture. Biol. Reprod. 64, 904-909. doi:10.1095/BIOLREPROD64.3.904 
Sun, Q., Wu, G., Lai, L., Park, K., Cabot, R., Cheong, H., Day, B., Prather, R., and Schatten, H. (2001). Translocation of active mitochondria during pig oocyte maturation, fertilization and early embryo development in vitro. Reproduction 122, 155-163. doi:10.1530/REP.0.1220155

Sutton, M., Cetica, P., Beconi, M., Kind, K., Gilchrist, R., and Thompson, J. (2003). Influence of oocyte-secreted factors and culture duration on the metabolic activity of bovine cumulus cell complexes. Reproduction 126, 27-34. doi:10.1530/REP.0.1260027

Sutton-McDowall, M., Gilchrist, R., and Thompson, J. (2005). Effect of hexoses and gonadotrophin supplementation on bovine oocyte nuclear maturation during in vitro maturation in a synthetic fluid medium. Reprod. Fertil. Dev. 17, 407-415. doi:10.1071/RD04135

Sutton-McDowall, M., Gilchrist, R., and Thompson, J. (2010). The pivotal role of glucose metabolism in determining oocyte developmental competence. Reproduction 139, 685-695. doi:10.1530/REP-09-0345

Takahashi, Y., and First, N. (1992). In vitro development of bovine one-cell embryos: influence of glucose, lactate, pyruvate, amino acid and vitamins. Theriogenology 37, 963-978. doi:10.1016/0093-691X(92) 90096-A
Tarkowski, A. K. (1966). An air-drying method for chromosome preparations from mouse eggs. Cytogenetics 5, 394-400. doi:10.1159/ 000129914

Trinder, P. (1969). Determination of glucose in blood using glucose oxidase with an alternative oxygen acceptor. Ann. Clin. Biochem. 6, 24-27.

Wakefield, S., Lane, M., Schulz, S., Hebart, M., Thompson, J., and Mitchell, M. (2007). Maternal supply of omega-3 polyunsaturated fatty acids alter mechanisms involved in oocyte and early embryo development in the mouse. Am. J. Physiol. Endocrinol. Metab. 294, E425-E434. doi:10.1152/AJPENDO.00409.2007

Yu, Y., Dumollard, R., Rossbach, A., Lai, A., and Swann, K. (2010). Redistribution of mitochondria leads to burst of ATP production during spontaneous mouse oocyte maturation. J. Cell. Physiol. 1, 672-680.

Zuelke, K., and Brackett, B. (1992). Effects of luteinizing hormone on glucose metabolism in cumulus-enclosed bovine oocytes matured in vitro. Endocrinology 131, 2690-2696. doi:10.1210/EN.131.6.2690 